\title{
RÉPARTITION SPATIO-TEMPORELLE DE LA GRANDE ALOSE ALOSA ALOSA (LINNÉ, 1766) ET DE L'ALOSE FEINTE ALOSA FALLAX (LACÉPĖDE, 1803) DANS LE GOLFE DE GASCOGNE.
}

\author{
C. TAVERNY (1), P. ELIE (1)
}

(1) Cemagref, Centre de Bordeaux, Unité de Recherche Ressources Aquatiques Continentales, 50 avenue de Verdun, 33612 CESTAS Cedex, France.

\section{RÉSUMÉ}

Ce travail sur la distribution en milieu marin de la grande alose Alosa alosa et de l'alose feinte Alosa fallax, au niveau du plateau continental océanique français de l'Atlantique Ouest, s'est appuyé sur les données de captures collectées pendant près de 3 ans, entre août 1986 et mars 1989, par trois types de campagnes expérimentales de chalutage menées par l'IFREMER (Institut Français de Recherche pour l'Exploitation de la Mer). Au vu de l'aire échantillonnée qui s'étend de l'île d'Ouessant jusqu'au Gouf de Capbreton (latitudes $48^{\circ} 30^{\prime} \mathrm{N}-43^{\circ} 45^{\prime} \mathrm{N}$ ) de la côte jusqu'à plus de $700 \mathrm{~m}$ de profondeur au large (longitudes $4^{\circ} \mathrm{W}-1^{\circ} 20^{\prime} \mathrm{W}$ ), Alosa fallax fréquente particulièrement les zones de moins de $50 \mathrm{~m}$ de profondeur et ne dépasse pratiquement pas l'isobathe des $100 \mathrm{~m}$ contrairement à Alosa alosa qui se répartie de manière plus uniforme jusqu'à des fonds de plus de $100 \mathrm{~m}$. Leur distribution est de type agrégatif, en panache autour des embouchures des principaux bassins versants d'accueil (Gironde, Loire). Chez les deux espèces, et particulièrement pour l'alose feinte, les individus de plus grande taille sont rencontrés dans les zones les plus profondes. L'amplitude des âges varie de 2 à 5 ans chez $A$. alosa et de 2 à 6 ans chez $A$. fallax. Sur l'ensemble des campagnes, 89 à $95 \%$ des individus $A$. alosa appartiennent à la classe d'âge des 2 et 3 ans et 85 à $92 \%$ des $A$. fallax ont entre 3 et 4 ans. La quasi absence de jeunes $A$. fallax âgés de 2 ans confirme une inféodation plus forte aux milieux estuariens à ce stade. En comparant les caractéristiques biométriques d'A. alosa âgées de 2 ans provenant d'une part de l'estuaire de la Gironde et d'autre part du milieu marin, il apparaît que le coefficient de condition des premiers est significativement plus élevé. Le temps d'adaptation nécessaire au passage d'un milieu estuarien riche en proies à un milieu marin offrant de nouvelles conditions peut expliquer une perte d'embonpoint.

Mots-clés : distribution géographique, mer, Alosa alosa, Alosa fallax, écologie. 


\title{
SPATIO-TEMPORAL DISTRIBUTION OF ALLIS SHAD (ALOSA ALOSA L.) AND TWAITE SHAD (ALOSA FALLAX L.) IN THE BAY OF BISCAY.
}

\begin{abstract}
This study of the offshore distribution of allis shad Alosa alosa and twaite shad Alosa fallax throughout the French continental shelf area of the Atlantic ocean is based on catch data collected over the 3 - year period August 1986-March 1989, during bottom trawl surveys performed by the French research institute for exploitation of the sea (IFREMER). The survey area extends from Ouessant island to "Gouf de Capbreton " (48 $30^{\prime}$ N $\left.43^{\circ} 45^{\prime} \mathrm{N}\right)$ down to a depth of $700 \mathrm{~m}\left(4^{\circ} \mathrm{W}-1^{\circ} 20^{\prime} \mathrm{W}\right)$. Alosa fallax is distributed primarily at water depths $<50 \mathrm{~m}$. Contrary to Alosa alosa which ranges in water deeper than $100 \mathrm{~m}$, A. fallax doesn't occur over this depth. Each distribution of allis shad and twaite shad is aggregated and located in the river mouths of the most important watersheds (Gironde, Loire). For the two species and especially for twaite shad the largest fish are encountered in the deeper zone. The ages of $A$. alosa and $A$. fallax range from 2 to 5 and 2 to 6 years old. From all the campaigns, most of the allis shads ( 89 to $95 \%$ ) are 2 and 3 years old and most of the twaite shads ( 85 to $92 \%$ ) are 3 and 4 years old. Young twaite shad seem to be more dependent on estuary environment. The coefficient of condition of two years old allis shads caught in the estuary is significantly higher than for those captured at sea. The necessary adaptation period to migrate from the estuary to the marine new habitat, can explain a loss of condition.
\end{abstract}

Key-words : distribution, sea, Alosa alosa, Alosa fallax, ecology.

\section{INTRODUCTION}

L'alose vraie (Alosa alosa) et l'alose feinte (Alosa fallax) sont des poissons anadromes dont l'aire de répartition géographique s'étend sur toutes les côtes atlantiques Ouest (entre $28^{\circ}$ et $60^{\circ}$ Lat $N$ ) et jusqu'en Méditerranée. L'aspect général de leur corps est très semblable, avec, cependant, une forme plus allongée et cylindrique chez $A$. fallax. Le critère de distinction absolu retenu est le nombre de branchiospines (SPILLMAN, 1961; WHEELER, 1969 ; BAUCHOT et PRAS, 1980 ; TAVERNY, 1991).

Au stade adulte, la taille d'A. alosa peut atteindre $700 \mathrm{~mm}$ et un poids maximum de $4 \mathrm{~kg}$. Les caractéristiques biométriques de cette espèce se rapprochent de celle de la plus grande des aloses américaines A. sapidissima $(760 \mathrm{~mm}->3.5 \mathrm{~kg})$. Les proportions d'A. fallax restent elles plus modestes (550 mm - $2 \mathrm{~kg}$ ).

En France, l'alose vraie est celle qui subit la pression de pêche la plus importante durant sa migration de reproduction dans les fleuves. En 1981, elle se classait au $13^{\text {ème }}$ rang en valeur d'apport parmi les 20 premières ressources halieutiques du golfe de Gascogne (ELIE et RIGAUD, 1984).

La phase de vie marine des deux espèces a été peu étudiée (ELIE, 1985 ; ELIE, 1990). En mer Méditerranée, l'alose feinte reste à proximité des estuaires et ne gagne guère la haute mer (ROULE, 1925 ; LAHAYE, 1962). Au large des côtes marocaines, les aloses vraies âgées de 2 à 8 ans séjournent à proximité de l'upwelling estival situé vers Essaouira et Agadir, entre 30 et $150 \mathrm{~m}$ de profondeur (LAROCHE, 1985).

En 1989, QUERO et al. mettaient en évidence que la répartition chez les deux espèces restait côtière dans le golfe de Gascogne avec des abondances plus fortes 
d'A. alosa en début d'hiver au sud de la Bretagne et au printemps entre les côtes vendéenne et landaise $\left(47-48^{\circ} \mathrm{N} / 2-4^{\circ} \mathrm{W}, 45-46^{\circ} \mathrm{N} / 2^{\circ} \mathrm{W}\right)$.

Les informations restent malgré tout générales, contrairement à celles qui traitent de la vie des aloses en milieu fluvial (KARTAS, 1981 ; CASSOU-LEINS et CASSOU-LEINS, 1981 ; DOUCHEMENT, 1981; EIRAS, 1981; DAUTREY et LARTIGUE, 1983 ; BOISNEAU et MENNESSON-BOISNEAU, 1990).

La migration de reproduction des aloses commence dans les estuaires au mois de mars et se termine à la fin des mois de juin-juillet (ANONYME, 1978 ; CASSOU-LEINS et CASSOU-LEINS, 1981). Le retour vers la rivière natale semble bien marqué chez les deux espèces (DOUCHEMENT, 1981). Les bassins français de la façade atlantique qui les accueillent à un niveau plus ou moins fort sont la Vilaine, la Loire, la Charente, la GirondeGaronne-Dordogne, et l'Adour.

Nés respectivement vers les mois de mai-juin et juin-juillet, les jeunes de l'année (YOY) dévalent de leurs zones de frayère jusqu'à la mer. Dans l'estuaire de la Gironde, les YOY $A$. fallax sont abondants dès août-septembre. Ils gagnent progressivement les zones de plus en plus salées au fur et à mesure de leur croissance. Ils se concentrent préférentiellement dans un premier temps en secteur oligo-mésohalin. Leur pénétration dans l'estuaire est lente. Au contraire, les juvéniles $A$. alosa occupent très rapidement l'ensemble de l'estuaire quelle que soit leur taille. Ils sont abondants essentiellement durant les mois de novembre-décembre.(ANONYME, 1979 ; BOIGONTIER et MOUNIÉ, 1984 ; TAVERNY, 1988). Les alosons évoluent préférentiellement en surface, le phénomène étant plus marqué chez $A$. fallax. Dès que les conditions de milieu deviennent trop limitantes (température $<5^{\circ} \mathrm{C}$, salinité $<0,5 \%$ ), les juvéniles gagnent le milieu marin. Dès le retour de conditions de milieu à nouveau favorables, les alosons sont susceptibles de retourner dans l'estuaire interne (TAVERNY, 1991). En période de montaison, des aloses de 2 et 3 ans peuvent être observées en eau douce parmi les géniteurs (BOISNEAU et MENNESSON-BOISNEAU, 1990 ; TAVERNY, 1991).

Cet article apporte des informations sur la différence de répartition spatiale entre les deux espèces d'aloses en milieu marin ainsi que sur l'amplitude du domaine occupé par des jeunes aloses de même cohorte.

\section{MÉTHODES}

\section{Aire d'étude}

Les prélèvements en milieu marin ont été réalisés sur le plateau continental du golfe

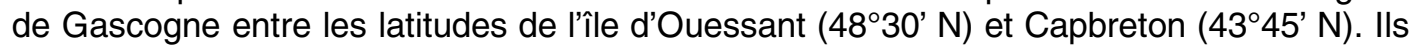
se répartissent de la zone littorale jusqu'à plus de $700 \mathrm{~m}$ de profondeur (Figure 1). $\left.0^{\circ} 41^{\prime} \mathrm{W}\right)$.

Les échantillons en milieu estuarien ont été effectués dans la Gironde $\left(45^{\circ} 15^{\prime} \mathrm{N} /\right.$ 


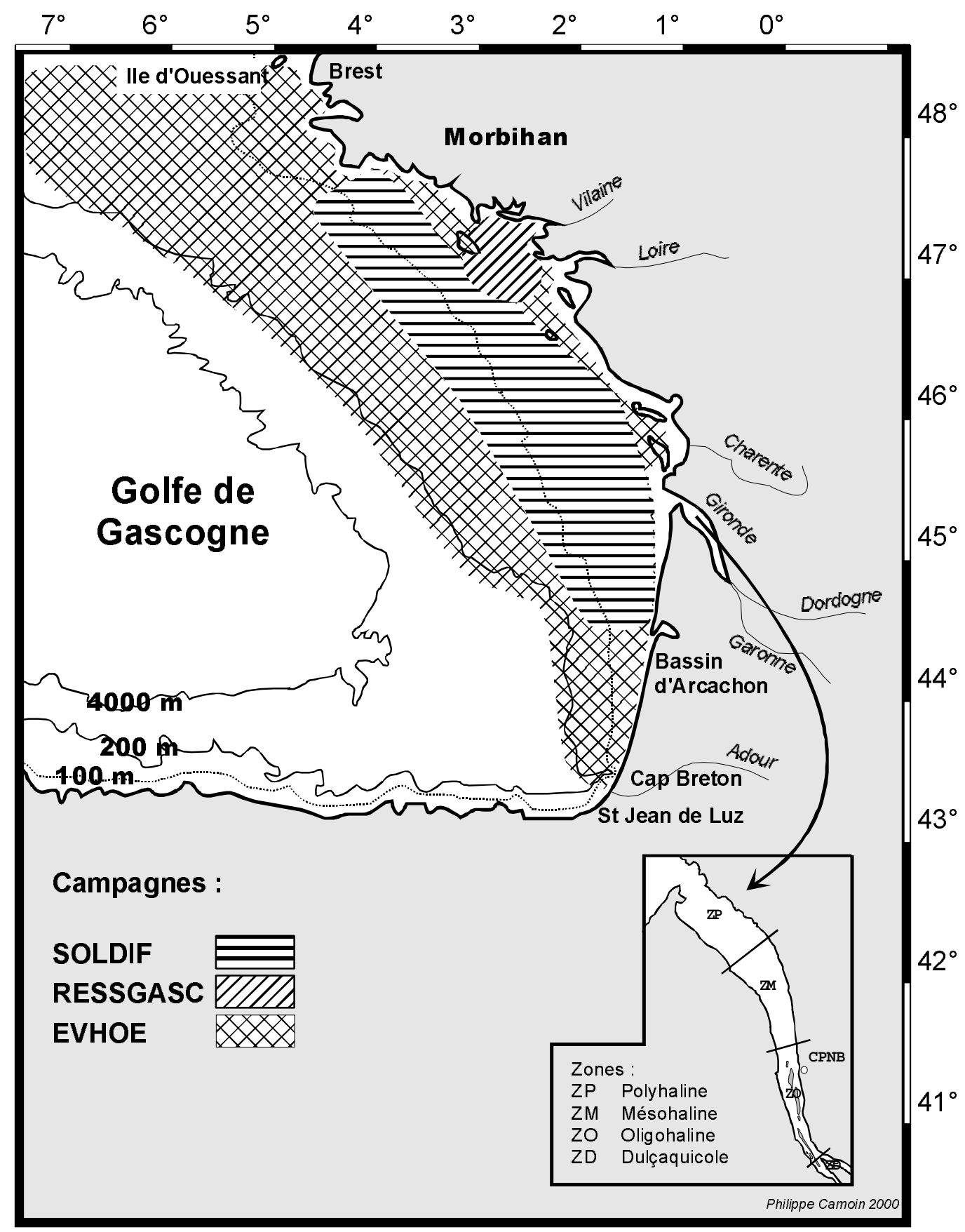

Figure 1

Aire de suivi de l'état des stocks d'espèces commerciales par chalutage de I'IFREMER entre 10 et plus de $700 \mathrm{~m}$ de profondeur de l'île d'Ouessant (Lat. $\left.48^{\circ} 30^{\prime} N\right)$ jusqu'au gouf de Capbreton $\left(43^{\circ} 45^{\prime} N\right)$ et situation du centre de production nucléaire de Blayais (CPNB) dans l'estuaire de la Gironde.

Figure 1

IFREMER bottom trawl surveys of commercial fish stocks area between depths of 10 and $700 \mathrm{~m}$, Ouessant island $\left(48^{\circ} 30^{\prime} \mathrm{N}\right)$ to gouf de Capbreton $\left(43^{\circ} 45^{\prime} \mathrm{N}\right)$ Blayais Nuclear Power Plant (CPNB) location in the Gironde estuary. 


\section{Collections}

En mer

3 séries de campagnes de chalutage menées par l'IFREMER* entre août 1986 et mars 1989 sont à l'origine de la collection d'aloses, captures accessoires de pêches scientifiques ayant pour objectifs principaux le suivi de l'état des stocks d'espèces commerciales (campagnes RESSGASC, SOLDIF), et plus largement l'évaluation des ressources halieutiques du Golfe de Gascogne (campagnes EVHOE) (Tableau I).

\section{Tableau I}

Résumé des campagnes de chalutage menées par I'IFREMER - 1986-1989 (R RESSGASC ; E EVHOE ; S SOLDIF).

\section{Table I}

Summary of IFREMER trawl campaigns - 1986-1989 (R RESSGASC ; E EVHOE ; S SOLDIF).

\begin{tabular}{|c|c|c|c|c|c|c|}
\hline \multirow[t]{2}{*}{ Saison } & \multicolumn{3}{|c|}{$\begin{array}{l}\text { Nombre de } \\
\text { campagnes }\end{array}$} & \multicolumn{2}{|c|}{$\begin{array}{l}\text { Nombre de } \\
\text { stations }\end{array}$} & \multirow[t]{2}{*}{ Dates incluses } \\
\hline & $\mathrm{R}$ & 2 & & 84 & & \\
\hline \multirow[t]{3}{*}{ Printemps } & $E$ & 1 & 4 & 136 & 239 & 13 avril au 7 juin \\
\hline & $S$ & 1 & & 19 & & \\
\hline & $\mathrm{R}$ & 3 & & 142 & & \\
\hline \multirow[t]{3}{*}{ Eté } & E & 0 & 5 & 0 & 190 & 4 juill. au 29 sept. \\
\hline & $S$ & 2 & & 48 & & \\
\hline & $\mathrm{R}$ & 3 & & 116 & & \\
\hline \multirow[t]{3}{*}{ Automne } & E & 2 & 6 & 285 & 420 & 7 oct. au 9 déc. \\
\hline & $S$ & 1 & & 19 & & \\
\hline & $\mathrm{R}$ & 3 & & 85 & & \\
\hline \multirow[t]{2}{*}{ Hiver } & $E$ & 0 & 5 & 0 & 167 & 15 janv. au 11 mars \\
\hline & $S$ & 2 & & 82 & & \\
\hline Total & & & 20 & & 1016 & \\
\hline
\end{tabular}

Les pêches ont été menées selon un échantillonnage stratifié au hasard :

- depuis Le Morbihan (Lat. $48^{\circ} \mathrm{N}$ ) jusqu'au nord du bassin d'Arcachon entre $11 \mathrm{~m}$ et l'isobathe $100 \mathrm{~m}$ (Lat. $45^{\circ} \mathrm{N}$ ) (campagnes RESSGASC). Ces zones sont celles fréquentées par les chalutiers professionnels du large (Figure 1). L'engin utilisé est un chalut de fond type vendéen de $25 \mathrm{~m}$ de longueur de corde de dos avec une maille étirée de $55 \mathrm{~mm}$ pour le cul du chalut. L'effort de pêche par trait est dans la majorité des cas de $120 \mathrm{mn}$.

- au large des estuaires de la Vilaine et de la Loire (autour $47^{\circ} 17^{\prime} \mathrm{N}$ ) (campagnes SOLDIF). Elles se poursuivent au-delà de la zone littorale jusqu'au niveau de Belle-lle

* Institut Français de Recherche pour l'Exploitation de la Mer 
$\left(3^{\circ} 10^{\prime} \mathrm{W}\right)$ (Figure 1). Un chalut de fond de $25 \mathrm{~m}$ d'ouverture à double poche $(20 \mathrm{~mm}$ en maille étirée pour le cul) et un chalut à perche de $3 \mathrm{~m}$ d'ouverture (1 $\mathrm{mm}$ pour le cul) sont utilisés sur les zones de nourriceries.

- de Brest $\left(48^{\circ} 30^{\prime} N\right)$ jusqu'à Capbreton $\left(43^{\circ} 45^{\prime} N\right)$ depuis la côte vers 15 m jusqu'à $760 \mathrm{~m}$ (campagnes EVHOE) (Figure 1). Les chalutages sont bisannuels. L'engin de pêche est un chalut de fond à grande ouverture verticale. La corde de dos mesure $36 \mathrm{~m}$ et le bourrelet $47 \mathrm{~m}$. Le maillage étiré du cul du chalut est de $50 \mathrm{~mm}$ doublé intérieurement par une poche de $40 \mathrm{~mm}$. L'effort de pêche par trait est de $30 \mathrm{mn}$.

Les aloses pêchées étaient conservées par congélation directement sur le bateau. Les relevés de positionnement des stations (bathymétrie, latitudes / longitudes) enregistrées uniquement pour les campagnes RESSGASC et EVHOE ont été récupérées.

\section{En estuaire}

Dans le cadre d'une évaluation de la qualité du milieu estuarien de la Gironde et d'une éventuelle répercussion sur les stocks de juvéniles d'alose dévalants, l'influence de la pression mécanique du centre de production nucléaire de Blayais (CPNB) a été étudiée de manière hebdomadaire d'août 1985 à décembre 1988.

Cette usine est située dans une zone à dominante oligohaline (pK 53) et fonctionne en circuit ouvert (Figure 1). Elle pompe un maximum de $168 \mathrm{~m}^{3} / \mathrm{s}$ d'eau dans l'estuaire grâce à deux prises immergées situées à $400 \mathrm{~m}$ de la rive. L'eau acheminée vers le circuit de refroidissement passe d'abord dans 8 tambours filtrants à maille carrée de $3 \mathrm{~mm}$ de côté. Les organismes et les détritus sont décolmatés des tamis par des jets d'eau. L'engin d'échantillonnage, un filet poche, était placé dans chacune des 2 canalisations collectant les eaux de lavage des tambours filtrants. Long de $4 \mathrm{~m}$, le filet a une maille carrée de $4 \mathrm{~mm}$ terminé par une nappe de maille $1 \mathrm{~mm}$.

Les individus ont été conservés dans du formaldéhyde (10\%). Pour les besoins de cet article, seules les jeunes aloses récoltées durant la période décembre 1986 à mars 1987 et âgées de 2 ans sont retenues.

\section{Méthodes de laboratoire et calculs}

Afin de déterminer l'effet des deux modes de conservation sur la longueur totale (LT) et poids total (WT), des aloses fraîches ont été mesurées au $\mathrm{mm}$ et $\mathrm{g}$ près puis formolées ou congelées, et remesurées après formolisation et décongélation.

La conservation par le formol ne donne pas de différence significative (NS) au niveau du poids mais produit un écart sur la taille hautement significatif $\left(\mathrm{S}^{\star * *}\right)$ (test $t$ de Student). L'erreur vraisemblable maximum est de l'ordre de $2 \mathrm{~mm}$; elle est jugée suffisamment faible en regard de la précision de la mesure (Tableau II).

La congélation / décongélation affectent de manière très hautement significative à la fois le poids et la longueur des aloses (Tableau III).

En conséquence, les valeurs biométriques des aloses congelées ont été recalculées pour un état frais (Tableau IV). 


\section{Tableau II}

Etude de l'effet de la formolisation sur la taille $(\mathrm{mm})$ et le poids $(\mathrm{g})$ des juvéniles Alosa - Test t sur séries appariées.

\section{Table II}

Formalin effect study on young shad total length ( $\mathrm{mm}$ ) and total weight (g) Test $t$ on paired series.

\begin{tabular}{|c|c|c|c|c|c|}
\hline & \multicolumn{2}{|c|}{ A. alosa } & \multicolumn{2}{|c|}{ A. fallax } & \multirow{2}{*}{$\begin{array}{l}\text { limite de confiance de l'erreur } \\
\text { moyenne vraisemblable pour } \\
\text { un degré de } 99 \% \\
0.5 \text { à } 1.5\end{array}$} \\
\hline $\mathrm{Lt}$ & $n=67$ & $P<0.001$ & $n=40$ & $P<0.001$ & \\
\hline $\mathrm{mm}$ & 42 à 85 & $S^{* * *}$ & 56 à 102 & $\mathrm{~S}^{\star \star \star}$ & \\
\hline $\mathrm{Wt}$ & $n=39$ & $P<0.2$ & $n=31$ & $P<0.2$ & \pm 0.05 \\
\hline$g r$ & 0.5 à 2.85 & NS & 1.10 à 8.00 & NS & \\
\hline
\end{tabular}

\section{Tableau III}

Etude de l'effet de la congélation sur la taille et le poids des adultes Alosa, cas d'A. fallax - Test $t$ sur séries appariées.

Table III

Freezing effect study on shad total length $(\mathrm{mm})$ and total weight, case of A. fallax - Test t on paired series.
A. fallax $-n=21$
limite de confiance de l'erreur moyenne vraisemblable pour un degré de $99 \%$

$\begin{array}{cccc}\text { Lt } & \begin{array}{c}367 \mathrm{~mm} \\ (282 \text { à } 449)\end{array} & \mathrm{P}<0.001 & 0.5 \text { à } 11.5 \mathrm{~mm} \\ & & \mathrm{~S}^{* * *} & \\ \text { Wt } & 411 & \mathrm{P}<0.001 & 0.6 \text { à } 16,1 \mathrm{~g} \\ & (184 \text { à 692) } & \mathrm{S}^{* * *} & \end{array}$




\section{Tableau IV}

Formules de régression entre la taille (LT) et le poids (WT) d'A. alosa et d'A. fallax à l'état frais et préservées.

\section{Table IV}

Length (TL) and weight (TW) regression formulas between fresh and preserved A. alosa and $A$. fallax.

\begin{tabular}{|c|c|}
\hline A. alosa & A. fallax \\
\hline Lt frais $=3.206+1.007$ Lt cong & Lt frais $=8.944+0.992 \mathrm{Lt}$ cong \\
\hline$r=0.9963$ & $r=0.9987$ \\
\hline étendue : 214 à 540 mm & étendue : 276 à $445 \mathrm{~mm}$ \\
\hline Wt frais $=-2.825+1.016 \mathrm{Wt}$ cong & Wt frais $=-0.978+1.023 \mathrm{Wt}$ cong \\
\hline$r=0.9995$ & $r=0.9998$ \\
\hline étendue : 87.2 à $1670.0 \mathrm{~g}$ & étendue : 184.2 à $692.4 \mathrm{~g}$ \\
\hline
\end{tabular}

L'âge des aloses de provenance maritime fut estimé uniquement à partir des otolithes en raison de l'effet abrasif des traits de pêche sur les écailles. Les sagitta du genre Alosa restant relativement petits et minces jusqu'au stade adulte, l'examen direct face convexe vers l'examinateur peut être effectué sous microscope binoculaire stéréoscopique (grossissement 40x, fond noir, en lumière réfléchie).

Chez les deux espèces, la période de reproduction est connue et s'étend sur un court laps de temps (CASSOU-LEINS et CASSOU-LEINS, 1981; DOUCHEMENT, 1981 ; DAUTREY et LARTIGUE, 1983). Aussi, des mois de naissance théoriques peuvent être retenus : $A$. fallax : juin - $A$. alosa : juillet (TAVERNY, 1988).

L'âge moyen est calculé selon la formule de FAHY (1978) :

$$
\mathrm{A}=\Sigma \mathrm{niAi} / \mathrm{N}
$$

(où Ai est l'âge de la classe i, ni le nombre d'individus dans la classe i, $\mathrm{N}$ le nombre total)

L'indice de dispersion I mesure l'agrégation. II est utilisé pour les traits de pêche réalisés en mer. Les distributions sont assumées comme étant non dues au hasard quand I > 1 jusqu'à I = x (ELLIOT, 1977).

$$
\begin{gathered}
\mathrm{I}=\mathrm{s}^{2} / \mathrm{x} \\
\left(\mathrm{s}^{2} \text { variance } ; \mathrm{x}\right. \text { moyenne de l'échantillon) }
\end{gathered}
$$

Le coefficient de condition K de BECKMAN (1948) permet de comparer les sujets d'une même espèce. La relation allométrique longueur-poids variant peu au cours de la croissance, ce coefficient permet de suivre les variations de l'état physiologique du poisson par rapport à sa valeur spécifique normale (BAUCHOT et BAUCHOT, 1978).

$$
\mathrm{K}=10^{5} * \mathrm{Wt} / \mathrm{Lt}^{3} \quad(\mathrm{~W}(\mathrm{~g}) \text { et } \mathrm{Lt}(\mathrm{mm})) .
$$




\section{RÉSULTATS}

\section{Milieu marin}

Les campagnes RESSGASC et EVHOE ont permis d'échantillonner la quasi-totalité de l'aire du plateau continental français est atlantique. Les pêches qui se sont étagées de 11 à $760 \mathrm{~m}$ de profondeur ont permis de recueillir 282 grandes aloses et 226 aloses feintes. Malgré leurs caractéristiques d'échantillonnage différentes (puissance et effort de pêche) les deux campagnes ont été utilisées pour l'analyse de la fréquence des captures en relation avec la profondeur. Seules les captures supérieures ou égales à deux individus par trait de pêche ont été conservées. Les profondeurs des stations correspondantes s'étendent de 15 à $115 \mathrm{~m}$. La totalité des captures d'A. fallax et la plupart de celles d'A. alosa (78\%) se localisent à moins de $100 \mathrm{~m}$ (Figure 2$)$.

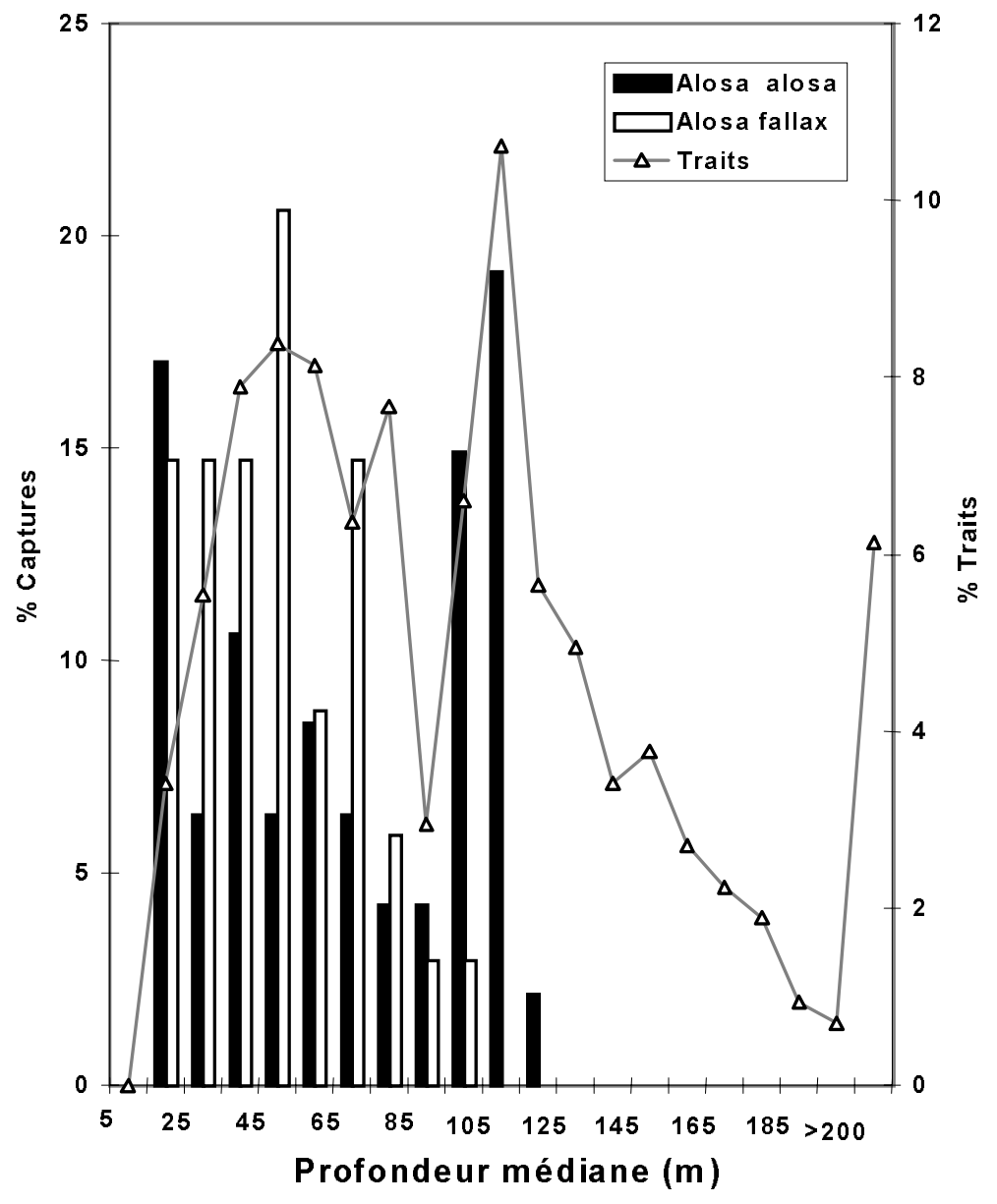

Figure 2

Distribution des fréquences des aloses capturées selon la profondeur pour 848 stations de surveillance, campagnes RESSGASC et EVHOE, 1986-1989 de l'île d'Ouessant jusqu'au gouf de Capbreton.

\section{Figure 2}

Frequency distribution (by median depth $(\mathrm{m})$ ) of shad captured within 848 survey stations, RESSGASC and EVHOE campaigns, 1986-1989 from Ouessant island to " gouf de Capbreton ». 
Les nombres de traits de chalut avec des captures de poissons ont été soumis pour chaque espèce et chaque strate de profondeur au test non paramétrique du $\chi^{2}$ (avec correction de la continuité de YATES) (Tableau V). Les nombres des captures attendues comparés à ceux des captures observés indiquent que la collecte est significativement plus forte à moins de $50 \mathrm{~m}$ pour $A$. fallax $\left(\mathrm{p}<10^{-3}\right)$. Elle est aussi significativement moins forte au delà de $100 \mathrm{~m}$ pour $A$. fallax et au delà de $150 \mathrm{~m}$ pour $A$. alosa $\left(\mathrm{p}<10^{-2}\right)$.

\section{Tableau V}

Intervalles de profondeur et traits de chalut associés à une capture supérieure ou égale à 2 aloses Alosa alosa $(A)$ et d'Alosa fallax $(F)$ pour les campagnes RESSGASC et EVHOE $\left({ }^{* *} p<0.01 ;{ }^{* * *} p<0.001\right)$.

\section{Table V}

Depth intervals and bottom trawls with only catches of 2 or more shad $(A$. alosa (A) - A. fallax (F)) during RESSGASC and EVHOE campaigns $\left(^{* *} p<0.01\right.$; $\left.{ }^{* * \star} p<0.001\right)$.

\begin{tabular}{|c|c|c|c|c|c|c|c|}
\hline \multirow{3}{*}{$\begin{array}{l}\text { Intervalle de } \\
\text { profondeur } \mathrm{p} \\
\text { en mètres }\end{array}$} & \multirow{3}{*}{$\begin{array}{c}\text { Traits de } \\
\text { pêche } \\
\%\end{array}$} & \multicolumn{6}{|c|}{ Quantités de traits avec $A$. alosa ou $A$. fallax } \\
\hline & & \multicolumn{2}{|c|}{ Observées } & \multicolumn{2}{|c|}{ Attendues } & \multicolumn{2}{|c|}{$\chi^{2}$} \\
\hline & & A & $\mathrm{F}$ & A & $\mathrm{F}$ & A & $\mathrm{F}$ \\
\hline $\mathrm{p}<51$ & 25 & 19 & 22 & 12 & 9 & 3.52 & $17.36^{\star \star \star}$ \\
\hline $50<p<101$ & 32 & 19 & 12 & 15 & 11 & 0.82 & 0.02 \\
\hline $100<p<151$ & 28 & 10 & 0 & 14 & 9 & 1.45 & $10.03^{\star \star}$ \\
\hline$p>150$ & 15 & 0 & 0 & 7 & 5 & $8.04^{\star *}$ & 6.05 \\
\hline Total & 100 & 48 & 34 & 48 & 34 & & \\
\hline
\end{tabular}

La présence des aloses est notée au moins depuis le Morbihan $\left(47-48^{\circ} \mathrm{N}\right)$ jusqu'à Capbreton $\left(44^{\circ} \mathrm{N}\right)$. Les aloses feintes sont plus fréquentes entre l'île de Ré $\left(46^{\circ} \mathrm{N}\right)$ et une latitude correspondant à celle des grands lacs aquitains $\left(45^{\circ} \mathrm{N}\right)$. Les deux espèces d'aloses s'éloignent peu des côtes (maximum 30 miles). Malgré une absence d'échantillonnage saisonnière systématique sur l'ensemble des strates du plateau continental pour certaines campagnes (EVHOE ; SOLDIF) certaines régions apparaissent plus fréquentées. Près de $90 \%$ des captures d'Alosa fallax se font au large de l'embouchure de la Gironde et des pertuis charentais (secteurs VIII et VI). Alosa alosa se répartit plus régulièrement depuis le large de la côte girondine jusqu'au Morbihan entre les latitudes $45^{\circ}$ et $48^{\circ} \mathrm{N}$ (Figure 3 ). 


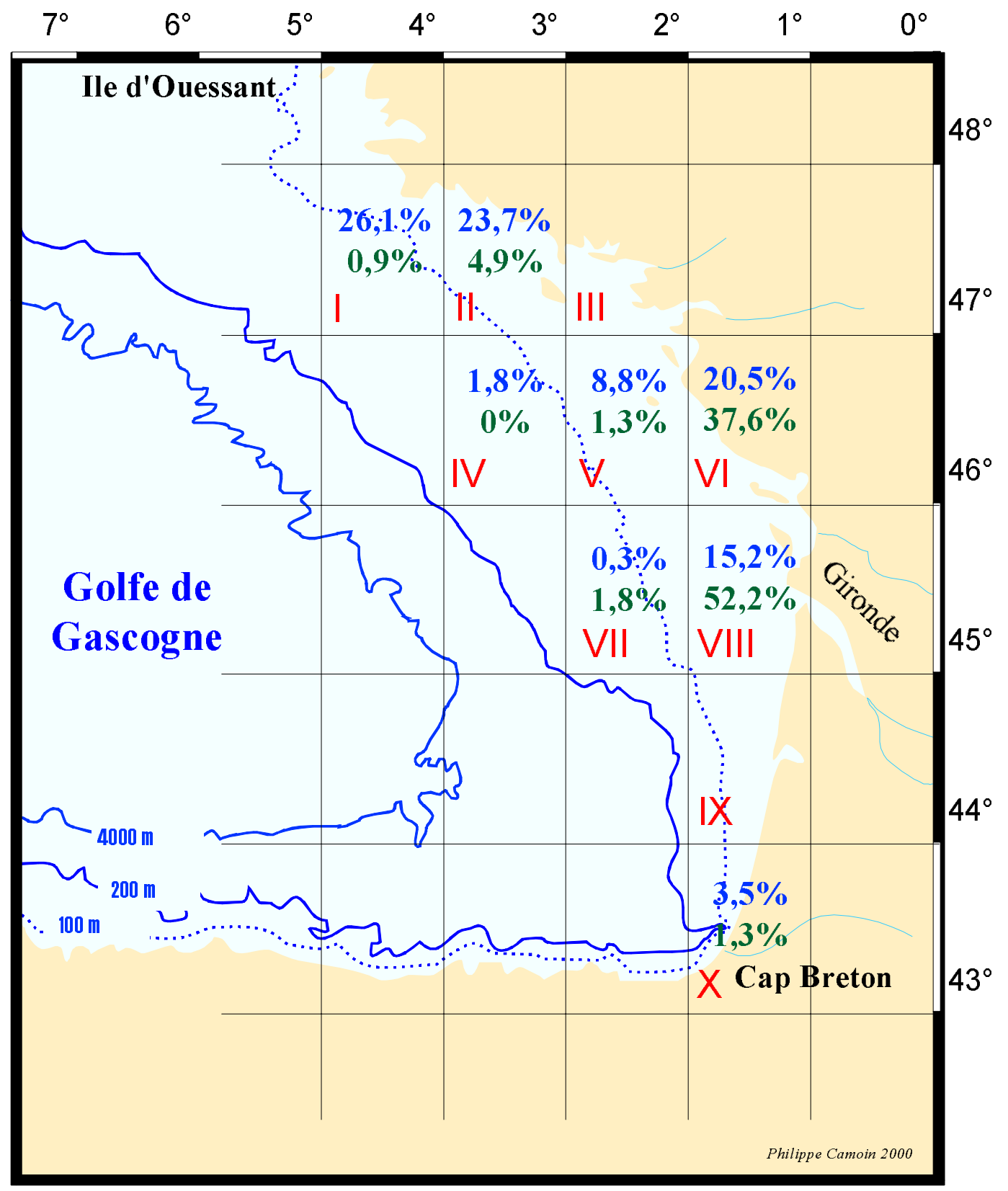

Figure 3

Répartition des prises d'A. alosa et d'A. fallax sur le plateau continental, campagnes RESSGASC et EVHOE, 1986-89, de l'île d'Ouessant jusqu'au gouf de Capbreton.

\section{Figure 3}

Location of $A$. alosa and $A$. fallax catches on continental plateau, RESSGASC and EVHOE campaigns, 1986-89, from Ouessant island to " gouf de Capbreton ". 
L'analyse des captures par campagne donne une distribution de type agrégatif autant chez l'alose vraie que chez l'alose feinte (respectivement dans $94 \%$ et $82 \%$ des cas étudiés) (Tableau VI).

\section{Tableau VI}

Indices de dispersion (I) en milieu marin toutes campagnes confondues (x moyenne ; SD déviation standard).

\section{Table VI}

Offshore dispersal index for RESSGASC, EVHOE and SOLDIF campaigns (x mean ; SD standard deviation).

\begin{tabular}{cccccccc} 
& \multicolumn{3}{c}{ A. alosa } & \multicolumn{3}{c}{ A. fallax } \\
\cline { 2 - 7 } & $\mathrm{x}$ & $\mathrm{SD}$ & $\mathrm{I}$ & $\mathrm{x}$ & $\mathrm{SD}$ & $\mathrm{I}$ \\
\hline Printemps & 0.46 & 5.93 & $\mathbf{1 2 . 8 9}$ & 0.15 & 0.63 & $\mathbf{4 . 2}$ \\
\hline Eté & 0.52 & 2.14 & $\mathbf{4 . 1 2}$ & 0.76 & 14.10 & $\mathbf{1 8 . 5 5}$ \\
\hline Automne & 0.09 & 0.73 & $\mathbf{8 . 1 1}$ & 0.12 & 0.59 & $\mathbf{4 . 9 2}$ \\
\hline Hiver & 0.43 & 1.55 & $\mathbf{3 . 6 0}$ & 0.19 & 0.47 & $\mathbf{2 . 4 7}$ \\
\hline
\end{tabular}

Sur l'ensemble des trois campagnes IFREMER, l'âge de 296 aloses vraies et 238 aloses feintes a été déterminé. L'amplitude des âges varie respectivement de 2 à 5 ans et de 2 à 6 ans. Selon les campagnes, 82 à $93 \%$ des individus $A$. alosa appartiennent à la classe d'âge des 2 et 3 ans ; environ $85 \%$ des $A$. fallax ont entre 3 et 4 ans et les âges extrêmes observés sont faiblement représentés (moins de $1 \%$ pour les 2 ans et de $4 \%$ pour les 6 ans) (Figure 4).

Chez les deux espèces, et particulièrement pour l'alose feinte, une corrélation hautement significative existe entre la profondeur, la taille et l'âge $\left(p<10^{-4}\right)$ des individus pêchés :
A. fallax
Bath $=0.2068 \times$ Lt -29.0169
$(n=230-r=0.6296)$
A. alosa
Bath $=0.2748 \times$ Lt -18.4169
$(n=286-r=0.4884)$
A. fallax
Bath $=12.9862 \times$ âge -12.5872
$(n=215-r=0.4344)$
A. alosa
Bath $=18.7107 \times$ âge +5.8118
$(n=277-r=0.3143)$

Le test non paramétrique de Kruskall-Wallis met en évidence, une différence très hautement significative entre les longueurs totales et les âges observés en fonction des catégories de profondeur ( $\mathrm{ddl}=3 ; \mathrm{p}<10^{-4}$ ) (Tableau VIII). 
campagnes RESSGASC

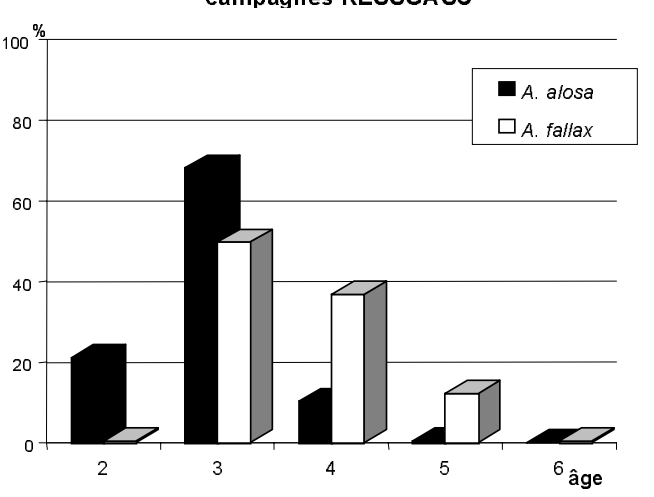

campagnes SOLDIF
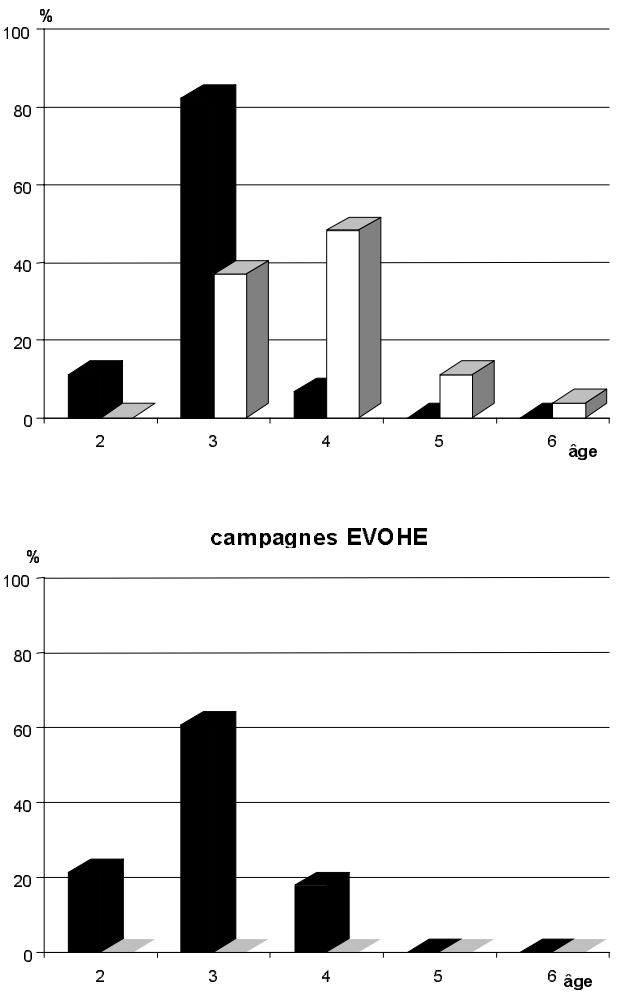

Figure 4

Distribution des fréquences de groupe d'âge pour $A$. alosa et $A$. fallax suivant chaque type de campagne IFREMER, 1986-1989, pour 1016 stations de surveillance de l'île d'Ouessant jusqu'au gouf de Capbreton.

\section{Figure 4}

Frequency distribution (by age-group) of $A$. alosa and $A$. fallax within each IFREMER campaigns, 1986-1989, for 1016 survey stations from Ouessant Island to " gouf de Capbreton ". 


\section{Tableau VII}

Tailles (LT en $\mathrm{mm}$ ) et poids (WT en g) moyens des $A$. alosa et des $A$. fallax, campagnes RESSGASC, EVHOE et SOLDIF confondues (moyenne-SD étendue - nombre).

Table VII

Mean lengths (TL in $\mathrm{mm}$ ) and weights (TW in g) of $A$. alosa and $A$. fallax collected during RESSGASC, EVHOE and SOLDIF campaigns (mean - SD - range number).

\begin{tabular}{|c|c|c|c|c|c|c|c|c|c|c|}
\hline & \multicolumn{2}{|c|}{2} & \multicolumn{2}{|c|}{3} & \multicolumn{2}{|c|}{4} & \multicolumn{2}{|c|}{5} & \multicolumn{2}{|c|}{6} \\
\hline & $\mathrm{Lt}$ & Wt & $\mathrm{Lt}$ & Wt & $\mathrm{Lt}$ & Wt & $\mathrm{Lt}$ & Wt & $\mathrm{Lt}$ & $\mathrm{Wt}$ \\
\hline \multirow[t]{4}{*}{ A. alosa } & 221 & 85 & 300 & 222 & 348 & 410 & 382 & 560 & & \\
\hline & 34 & 49 & 38 & 93 & 69 & 320 & - & - & & \\
\hline & $171-310$ & $32-230$ & 193-397 & $62-597$ & $249-538$ & $99-1684$ & - & - & & \\
\hline & 58 & 57 & 206 & 201 & 31 & 31 & 1 & 1 & & \\
\hline \multirow[t]{4}{*}{ A. fallax } & 169 & 28 & 261 & 140 & 347 & 327 & 391 & 429 & 473.5 & 616 \\
\hline & - & - & 41 & 77 & 45 & 144 & 47 & 171 & - & - \\
\hline & - & - & 201-397 & $58-597$ & $242-442$ & $83-683$ & $288-472$ & $168-786$ & $461-486$ & $580-652$ \\
\hline & 1 & 1 & 115 & 115 & 91 & 91 & 29 & 29 & 2 & 2 \\
\hline
\end{tabular}

\section{Tableau VIII}

Tailles (LT en $\mathrm{mm}$ ) et âges observés chez $A$. alosa (A) et $A$. fallax (F) selon la profondeur - campagnes RESSGASC.

\section{Table VIII}

Mean lengths (TL in $\mathrm{mm}$ ) and age of $A$. alosa and $A$. fallax in relation to depth RESSGASC campaign.

\begin{tabular}{|c|c|c|c|c|c|}
\hline \multirow[b]{2}{*}{$\begin{array}{l}\text { Intervalle de } \\
\text { profondeur p } \\
\text { (m) }\end{array}$} & & \multicolumn{2}{|c|}{ Longueur totale } & \multicolumn{2}{|c|}{ Age } \\
\hline & & A & $\mathbf{F}$ & A & $\mathbf{F}$ \\
\hline \multirow{3}{*}{$\mathrm{p}<26$} & $\mathrm{n}$ & 61 & 103 & 57 & 98 \\
\hline & $x$ & 252.4 & 264.1 & 2.8 & 3.2 \\
\hline & SD & 38.2 & 56.1 & 0.5 & 0.5 \\
\hline \multirow{3}{*}{$25<p<51$} & $\mathrm{n}$ & 65 & 75 & 66 & 70 \\
\hline & $x$ & 268.5 & 328.8 & 2.8 & 3.9 \\
\hline & SD & 53.7 & 46.4 & 0.6 & 0.8 \\
\hline \multirow{3}{*}{$50<p<101$} & $\mathrm{n}$ & 106 & 50 & 101 & 45 \\
\hline & $x$ & 292.2 & 368.5 & 2.9 & 4.1 \\
\hline & SD & 60.5 & 55.9 & 0.5 & 0.8 \\
\hline \multirow{3}{*}{$100<p<151$} & $\mathrm{n}$ & 52 & 2 & 51 & 2 \\
\hline & $x$ & 332.1 & 439.0 & 3.2 & 4.5 \\
\hline & SD & 52.6 & 8.5 & 0.5 & 0.7 \\
\hline
\end{tabular}




\section{Comparaison entre le milieu estuarien et marin}

Cas des aloses du groupe d'âge 2 ans

En milieu marin, $98 \%$ des aloses âgées de 2 ans faisaient partie de l'espèce A. alosa $(n=59)$. Aussi, seul le cas de cette espèce a pu être traité ici. Ces jeunes A. alosa furent pêchées entre $11 \mathrm{~m}$ et $97 \mathrm{~m}$.

Des alosons dans leur deuxième année furent récoltés d'août 1985 à décembre 1988 dans l'estuaire de la Gironde, en zones polyhalines et mésohalines.

Les individus de la cohorte 1985, suffisamment nombreux dans les deux types de milieu, ont permis une comparaison entre ceux observés en hiver 1986-1987 (TAVERNY, 1988) et ceux pêchés en février 1987 au large de la Gironde (campagne RESSGASC) entre 36 et $72 \mathrm{~m}$ de profondeur (Tableau IX).

\section{Tableau IX}

Caractéristiques biométriques des individus $A$. alosa de la cohorte 1985, à l'âge de 2 ans - hiver 1986-1987 (LT longueur totale en mm; WT poids total en $\mathrm{g}$; Kc coefficient de condition ; $n$ nombre d'individus ; $x$ moyenne ; s déviation standard; e étendue).

\section{Table IX}

Biometrics data of the $1985 \mathrm{~A}$. alosa cohort, at the age of 2 years - Winter 19861987 (TL total length in $\mathrm{mm}$; TW total weigth in $\mathrm{g}$; Kc condition index; $\mathrm{n}$ number ; $\mathrm{x}$ mean ; s standard deviation ; e range).

\begin{tabular}{ccccc} 
& & LT & WT & Kc \\
\cline { 3 - 5 } Milieu estuarien & $\mathrm{n}$ & 17 & 17 & 17 \\
Déc. 1986 à mars 1987 & $\mathrm{x}$ & 157.2 & 28.7 & 1.34 \\
& $\mathrm{~s}$ & 19.0 & 10.4 & 0.32 \\
& $\mathrm{e}$ & $125-202$ & $14.5-57.3$ & $1.14-2.52$ \\
Milieu marin (secteur 8) & $\mathrm{n}$ & 16 & 16 & 16 \\
Fév. 1987 & $\mathrm{x}$ & 201.9 & 53.2 & 1.16 \\
& $\mathrm{~s}$ & 17.2 & 17.2 & 0.11 \\
& $\mathrm{e}$ & $175-233$ & $29.7-85.5$ & $0.88-1.22$ \\
\hline
\end{tabular}

Par rapport aux individus observés en milieu estuarien, les valeurs en poids et en taille notées en milieu marin sont en moyenne plus fortes. Le test de Mann et Whitney confirme une différence hautement significative pour chacune des caractéristiques biométriques $(p<0.01$ ), le coefficient de condition indiquant un embonpoint plus élevé des individus résidant encore en estuaire. 


\section{DISCUSSION}

Pour les deux espèces, la représentation des individus dans leur deuxième année est très faible. De plus, les jeunes $A$. alosa ont été plus fréquemment observés que ceux d'A. fallax pour des localisations de pêche identiques.

Ceci pourrait laisser supposer d'une part que, dans leur distribution spatiale, les individus de 2 ans d'âge restent inféodés de façon plus étroite à la zone côtière, voire aux secteurs proches des embouchures estuariennes, et, d'autre part que ce phénomène serait plus marqué pour l'alose feinte.

La présence sur les arcs branchiaux de certaines aloses de copépodes parasites de l'espèce Clavellisa emarginata (Lerneapodidae), espèce marine, a prouvé que dès le groupe d'âge 1, les deux espèces sont capables d'effectuer des aller-retours entre le milieu estuarien et le milieu marin (TAVERNY, 1991). Les mêmes observations ont été faites avec des YOY $A$. sapidissima au niveau des estuaires de la façade atlantique-Est (CHITTENDEN, 1972a, 1972b ; MILSTEIN, 1981 ; LIMBURG, 1998), ces jeunes poissons gagnant les eaux océaniques dès que les eaux estuariennes présentent des températures et des salinités trop faibles.

De plus, $A$. fallax semble avoir un schéma de distribution plus dépendant des milieux estuariens. En période hivernale, les jeunes de 2 ans sont fréquemment observés dans l'estuaire de la Gironde contrairement aux aloses vraies rarement présentes (TAVERNY, 1991).

Cependant, les facteurs suivants peuvent fausser cette première lecture :

- une sous-exploration des fonds de moins de $20 \mathrm{~m}$ lors des campagnes RESSGASC et EVHOE ; ces fonds correspondent à la zone littorale, zone riche en nourriture (CASTEL, 1981; CLARK, 1974 ; KETCHUM, 1972) et qui réceptionne en premier les jeunes poissons dévalants.

- un échappement des jeunes aloses lié au maillage des chaluts utilisés durant les campagnes RESSGASC et EVHOE ; le phénomène pouvant être plus marqué dans le cas de l'alose feinte qui à âge équivalent est de plus petite taille que l'alose vraie.

Les jeunes $A$. alosa de 2 ans observés durant le même période montrent une capacité de distribution spatiale très large. Au vu de leurs caractéristiques biométriques, les individus plus petits profiteraient plus longtemps de la richesse faunistique du milieu estuarien. Chez ces jeunes formes, l'avantage serait d'une part de permettre de combler plus rapidement un retard de croissance et d'autre part de retarder l'entrée dans le milieu océanique (en comparaison moins riche en abondance faunistique), où des aptitudes physiques ou physiologiques seraient indispensables. Cette hypothèse reste bien sûr à confirmer.

Pour les deux espèces d'aloses, le comportement agrégatif observé dès les plus jeunes écophases que ce soit en milieu fluvial ou estuarien (TAVERNY, 1991) se poursuit en milieu marin.

Alosa fallax fréquente particulièrement les zones de moins de $50 \mathrm{~m}$ de profondeur et ne dépasse pratiquement pas l'isobathe des $100 \mathrm{~m}$ contrairement à Alosa alosa qui se répartit de manière plus uniforme jusqu'à des fonds de plus de $100 \mathrm{~m}$.

Les secteurs au large des embouchures des principaux fleuves, Gironde-Charente, Loire et Vilaine ressortent comme étant les plus «fréquentés » par les deux espèces. Cette observation pourrait être la conséquence d'une distribution en panache depuis les 
systèmes fluvio-estuariens d'accueil. II resterait cependant à appréhender dans toute leur ampleur les déplacements de ces poissons. La méthode par marquage peut s'avérer à cette fin efficace. Ainsi, grâce à plus de $8000 \mathrm{~A}$. sapidissima marquées à partir du fleuve St-Laurent, VLADIKOV $(1950,1956)$ a montré que cette espèce pouvait couvrir des distances de l'ordre de $2220 \mathrm{~km}$ sur près de 2 ans et descendre de $10^{\circ}$ en valeur de latitude. Grâce aux données de marquage-recapture (plus de 60000 ind. - $5.7 \%$ de retour), DADSWELL et al. (1987) sont arrivés à un constat de plus de $20000 \mathrm{~km}$ parcourus sur une durée moyenne de vie en mer de 5 ans ; les aires de migration extrêmes allant du Labrador (HODDER, 1966 ; HARE et MURPHY, 1974) à la Floride (WILLIAMS et BRUGER, 1972).

Les individus $A$. alosa de 6 et 7 ans, les plus âgés, capturés régulièrement lors des montaisons (CASSOU-LEINS et CASSOU-LEINS, 1981; DOUCHEMENT, 1981; BOISNEAU et MENNESSON-BOISNEAU, 1990 ; TAVERNY, 1991) n'apparaissent pas dans ces prélèvements réalisés en mer et les $A$. fallax de 6 ans sont à peine représentées. Les causes peuvent provenir du fait que la proportion des individus les plus âgés est moins importante, la probabilité de les capturer est alors plus faible. De plus, dès le printemps ces individus peuvent être essentiellement présents en milieu dulçaquicole en raison de leur migration génésique et en dehors de la période de reproduction, la répartition de ces individus pourrait être plus prononcée vers le large et vers des fonds plus importants. La corrélation observée entre la bathymétrie et l'âge des aloses va dans le sens de cette dernière hypothèse. Le suivi halieutique mené par ROULE (1925) indiquait la présence possible de l'alose vraie dans des coups de chalut effectués sur des fonds de plus de 200 et $300 \mathrm{~m}$ par des pêcheurs.

L'alose feinte apparaît inféodée à des zones moins profondes que les aloses vraies qui s'aventurent fréquemment jusqu'à des fonds situés autour de $100 \mathrm{~m}$. Ce résultat rappelle celui de NEVES (1981) qui a étudié pendant 16 ans, à l'aide de traits de chalut de fond, la distribution de deux espèces d'aloses américaines (côte Atlantique Est). II a ainsi mis en évidence qu'A. pseudoharengus se situait le plus souvent vers des fonds de 56 et $110 \mathrm{~m}$ et qu'A. aestivalis se situait entre 27 et $55 \mathrm{~m}$ (taille maximum des deux espèces $380 \mathrm{~mm}$ ). De plus, leurs mouvements verticaux semblaient suivre ceux du zooplancton dans la colonne d'eau.

La relation avec la température n'a pas été possible dans le cadre de cette étude car aucun relevé simultané n'a été réalisé lors des prélèvements IFREMER. Des travaux déjà réalisés dans ce domaine ont avancé que la thermique des masses d'eau maritimes semblait jouer un rôle essentiel dans la localisation des individus (NEVES et DEPRES, 1979 ; NEVES, 1981). Certains travaux plus récents modulent, cependant, l'importance de ce facteur (DADSWELL et al., 1987).

Il serait intéressant de poursuivre le suivi de la biologie des aloses en mer afin de compléter les observations réalisées, de vérifier les hypothèses émises et de tenter d'expliquer les schémas de leurs distributions en intégrant les facteurs abiotiques et biotiques.

\section{REMERCIEMENTS}

Nous voudrions remercier Messieurs DECAMPS, DESAUNAY, FOREST, GUICHET, LEAUTE, POULARD et QUERO de I'IFREMER pour la collecte des aloses en mer au cours des campagnes RESSGASC, EVHOE et SOLDIF et pour la conservation, le stockage de ces dernières à terre ainsi que pour la remise des caractéristiques des différentes campagnes. 


\section{BIBLIOGRAPHIE}

ANONYME, 1978. Annuaire statistique des pêches - F.A.O.. Captures et quantités débarquées, O.N.U.A.A. ed., 46, 372 p.

ANONYME, 1979. Etude halieutique de l'estuaire de la Gironde. Rapp. CTGREF div. ALA Bordeaux, $189 \mathrm{p}$.

BAUCHOT R., BAUCHOT M.L., 1978. Coefficient de condition et indice pondéral chez les téléostéens. Cybium, 3è série, 4, 3-16.

BAUCHOT M.L., PRAS A., 1980. Guide des Poissons Marins d'Europe. DELACHAUX et NIESTLÉ Eds., $427 \mathrm{p}$.

BECKMANN W.C., 1948. The length-weight relationship factors for conversions between standard and total length and coefficients of condition for seven Michigan fishes. Trans. Am. Fish. Soc., 75, 237-256.

BOIGONTIER B., MOUNIÉ D., 1984. Contribution à la connaissance de la dynamique de la macro-faune bentho-démersale et pélagique en Gironde. Tentatives et difficultés pour relativiser l'impact mécanique d'une centrale nucléaire Le Blayais (Gironde). Thèse Doctorat 3è cycle, Univ. de Toulouse / CEMAGREF div. ALA Bx, 491 p.

BOISNEAU P., MENNESSON-BOISNEAU C., 1990. Migration, répartition, reproduction, scalimétrie et caractéristiques biologiques des aloses (Alosa sp.) dans le bassin de la Loire. Thèse Doc. Univ. de Rennes I, 143 p.

CASSOU-LEINS F., CASSOU-LEINS J.J., 1981. Recherches sur la biologie et l'halieutique des migrateurs de la Garonne et principalement de l'Alose : Alosa alosa L. Thèse Doctorat 3è cycle, INP Toulouse, $382 \mathrm{p}$.

CASTEL J., 1981. Aspects de l'étude écologique du plancton de l'estuaire de la Gironde. Océanis, Doc. Océanogr., 6, 535-577.

CLARK J., 1974. Coastal ecosystems, Ecological considerations for management of the coastal zone. The Conservation Foundation, Washington, D.C., 178 p.

CHITTENDEN M.E. Jr., 1972a. Responses of young American shad (A. sapidissima) to low temperature. Trans. Am. Fish. Soc., 101 (4), 680-685.

CHITTENDEN M.E. Jr., 1972b. Salinity tolerance of young Blueback herring ( $A$. aestivalis). Trans. Am. Fish. Soc., 101 (1), 123-125.

DADSWELL M.J., MELVIN G.D., WILLIAMS P.J., THEMELIS D.E., 1987. Influences of origin, life history, and chance on the Atlantic coast migration of American Shad. In : Am. Fish. Soc. Symp. on Common strategies of anadromous and catadromous fishes, DADSWELL, KLAUDA, MOFFIT et al., Eds., 313-330.

DAUTREY R., LARTIGUE J.P., 1983. Recherches sur la migration des aloses (Alosa alosa) et des truites de mer (Salmo trutta) en Garonne (Site de Golfech). Thèse Doctorat 3è cycle, INP Toulouse, $212 \mathrm{p}$.

DOUCHEMENT C., 1981. Les Aloses des fleuves français, Alosa fallax L., 1803 et Alosa alosa L., 1758 ; biométrie, écobiologie : autonomie des populations. Thèse Doc. 3è cycle, Univ. Montpellier, $377 \mathrm{p}$.

EIRAS J., 1981. Contribuicao para o conhecimento da biologia de Alosa alosa L. Estudo de algumas modificaçoes somaticas, fisiologicas e bioquimibcas durante a migraçao anadroma no rio Douro. Thèse Doctorat 3è cycle, Univ. Porto, $228 \mathrm{p}$.

ELIE P., 1985. Les aloses en France : importance, état des connaissances et propositions d'un cadre pour la gestion de ces ressources. Note technique, Ministère de l'Environnement, Cemagref de Bordeaux, ALA, $26 \mathrm{p}$.

ELIE P., 1990. Les aloses (Alosa alosa L. et Alosa fallax Lac.). Assises de l'eau, groupe de travail "Gestion des Migrateurs Amphihalins », avril 1990, Ministère de l'Environnement, Cemagref de Bordeaux, ALBX, $12 \mathrm{p}$.

ELIE P., RIGAUD C., 1984. Etude de la population d'anguilles de l'estuaire et du bassin versant de la Vilaine. Pêche, biologie, écologie. Examen particulier de l'impact du barrage d'Arzal sur la migration anadrome (civelle). Rapp. Fac. Sc. Rennes / CEMAGREF div. ALA, Bordeaux / U.R.F.D.A.A.P.P. Bretagne-Maine-Normandie, $174 \mathrm{p}$. 
ELLIOT J.M., 1977. Some methods for the statistical analysis of samples of benthic invertebrates. Bio. Assoc. Sci. Publ., Fresh W. ed., 25, 160 p.

FAHY E., 1978. Variation in some biological characteristics of British seatrout. J. Fish. Biol., 13, 123-138.

HARE G.M., MURPHY H.P., 1974. First record of American Shad from labrador waters. J. Fish. Res. Board. Can., 31 (9), 1536-1537.

HODDER V.M., 1966. Two further records of the American Shad in New foundland waters. Trans. Am. Fish. Soc., 95, 228-229.

KARTAS F., 1981. Les Clupeidae de Tunisie - Caractéristiques biométriques et biologiques - Etude comparée des populations de l'Atlantique-Est de la Méditerranée. Thèse Doctorat 3è cycle, Univ. Tunis.

KETCHUM B.H., 1972. The water's edge - Critical problems of the coastal zone. MIT Press, Massachusetts, $393 \mathrm{p}$.

LAHAYE J., 1962. Etude histologique et histochimique de l'ovogénèse chez Alosa alosa III. L'ovocyte mûr et ses membranes. Soc. Sc. Nat. Phys. Maroc, 1, 29-32.

LAROCHE J., 1985. Contribution à la connaissance des peuplements de poissons demerssaux des côtes atlantiques marocaines du cap Spartel au cap Juby. Travaux Doc. Dév. Pêches, Maroc, 58, 27 p.

LIMBURG K.E., 1998. Anomalous migrations of anadromous herrings revealed with natural chemical tracers. Can. J. Fish. Aquat. Sci., 55, 431-437.

MILSTEIN C.B., 1981. Abundance and distribution of juvenile Alosa species of southern New Jersey. Trans. Am. Fish. Soc., 110, 306-309.

NEVES R.J., DEPRES L., 1979. The oceanic migration of american shad, Alosa sapidissima, along the atlantic coast. Fish. Bull., vol. 77 (1), 199-212.

NEVES R.J., 1981. Offshore distribution of alewife (Alosa pseudoharengus) and blueback herring (Alosa aestivalis) along the atlantic coast. Fish. Bull., 79 (3), 473-487.

QUERO J.C., DARDIGNAC J., VAYNE J.J., 1989. Les poissons du Golfe de Gascogne. Rapp. IFREMER / Mus. Nat. Hist. Nat., 229 p.

ROULE L., 1925. Poissons des eaux douces. P.U.F. ed., Paris, 69-83.

SPILLMAN J., 1961. Faune de France : 65 poissons d'eau douce. Féd. Fr. Soc. Sci. Nat. Off. Cent. Faunis., P. Chevalier, Paris, $301 \mathrm{p}$.

TAVERNY C., 1988. An attempt to estimate Alosa alosa and Alosa fallax juvenile mortality caused by three types of human activity in the Gironde estuary, 1985-1986. In : VAN DENSEN, STEINMETZ and HUQHES Eds. Proc. Symp. European Inland Fish. ADV. Comm., Göteborg, Sweden, 215-229.

TAVERNY C., 1991. Contribution à la connaissance de la dynamique des populations d'aloses (Alosa alosa et Alosa fallax) dans le système fluvio-estuarien de la Gironde - Pêche, biologie, écologie. Etude particulière de la dévalaison et de l'impact des activités humaines. Thèse Doctorat de l'Université, Université Bordeaux / CEMAGREF Bordeaux, 451 p. + Ann.

VLADYKOV V.D., 1950. Movements of Quebec shad Alosa sapidissima as demonstrated by tagging. Nat. Can., 77, 121-135.

VLADYKOV V.D., 1956. Distant recaptures of shad tagged in Quebec. Nat. Can., 83, 235249.

WHEELER A., 1969. Fish-life and pollution in the lower Thames : a review and preliminary report. Biol. Conserv., 2 (1), 25-30.

WILLIAMS R.O., BRUGER G.E., 1972. Investigations on American Shad in the St Johns river. Mar. Res. Lab., Florida Dep. Nat. Res., St Petersburg, Florida, Tech. Ser., 66, $49 \mathrm{p}$. 
